\title{
A direct and inverse problem for wave crests modelled by interactions of two solitons
}

\author{
Pearu Peterson ${ }^{1}$ \\ Department of Mechanics and Applied Mathematics, Institute of Cybernetics at \\ Tallinn Technical University, Akadeemia Rd. 21, 12618 Tallinn, Estonia \\ Fax: +372 6204161 Phone: +372 6204168 E-mail: pearu@ioc.ee \\ E. van Groesen \\ Faculty of Mathematical Sciences, University of Twente, P.O. Box 217, \\ 7500 AE Enschede, The Netherlands \\ E-mail:groesen@math.utwente.nl
}

\begin{abstract}
The paper addresses a new "inverse" problem for reconstructing the amplitudes of $2 \mathrm{D}$ surface waves from observation of the wave patterns (formed by wave crests). These patterns will depend on the amplitudes because of nonlinear effects. We show that the inverse problem can be solved when the waves are modelled by an equation that supports soliton solutions. Specifically, the explicit solution to the inverse problem is derived for two interacting solitons of the KP (Kadomtsev-Petviashvili) equation. As a prerequisite, the "direct" problem of two-soliton solutions is investigated, presented in such a way that generalizations to an arbitrary number of solitons can be done. In this investigation we give a new meaning to the concept of interaction soliton that makes it easier to write down the two-soliton solution and to describe the soliton interactions.
\end{abstract}

Key words: Wave pattern; Soliton interaction; Interaction soliton; Inverse problem.

PACS: 04.30.Nk; 03.40.Kf; 06.90.+v; 02.50.Sk

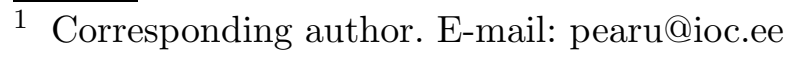

Preprint submitted to Elsevier Preprint

1 March 2000 


\section{Introduction}

The surface of a layer of fluid in motion can show a variety of continuously changing "patterns" formed by wave crests that diminish or increase in height, disappear, propagate, collide with other crests, etc. The translation from visual appearance to actual wave heights is not as easy as it may seem. To illustrate this, consider Figure 1 which is an animated snapshot of evolving surface waves where wave patterns are formed by wave crests. Points on a crest will not be, in general, points of maximum height, but rather saddle points: transverse (more or less perpendicular) to the crest they have maximal height, but along the crest, the height may be increasing, decreasing, or stationary. This makes it difficult, even when an explicit formula for a complicated surface elevation is given, to identify the collection of points that form a wave crest.

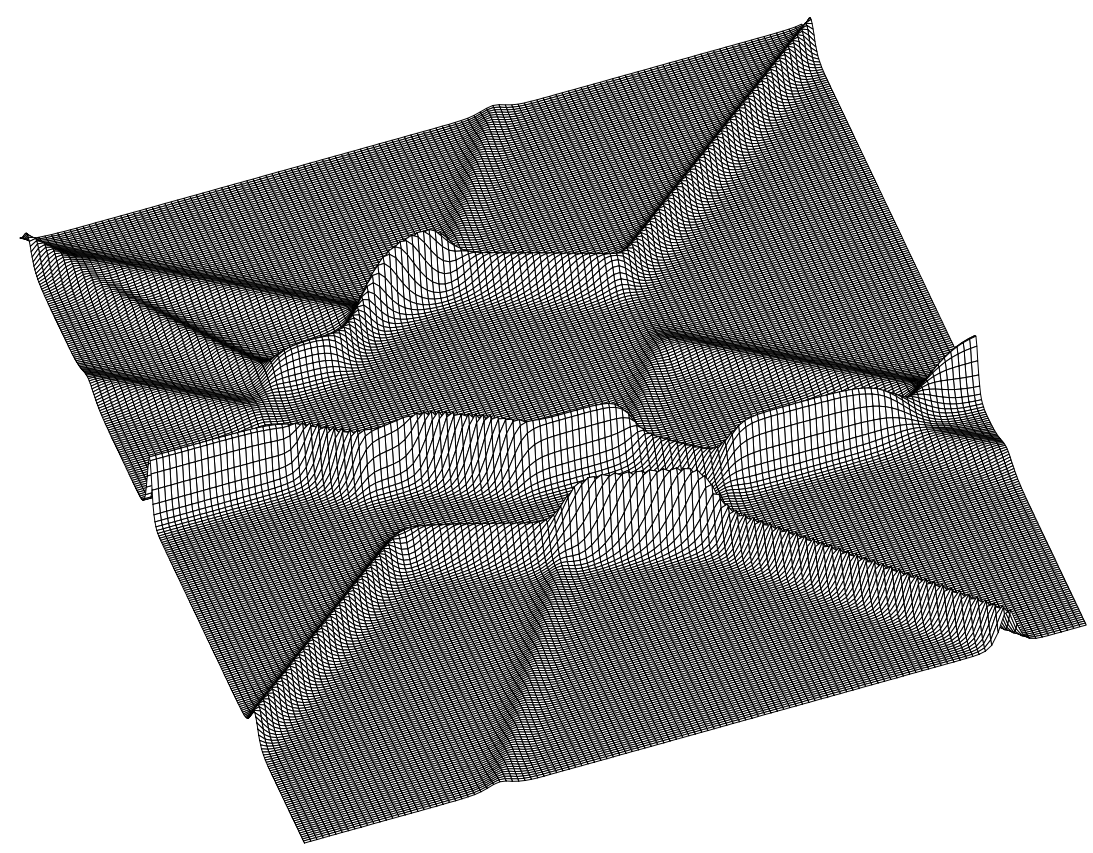

Fig. 1. Interaction of five waves modeled by solitons (abstract case, generated from the analytical formula of a five-soliton solution given by Hirota's bilinear formalism).

The motivation for the research presented in this and a successive paper found its origin in the paper of Hammack et al. [1], and in practical studies in hydrodynamic laboratories where waves in large basins are generated to test ships. The paper of Hammack et al. [2] shows photographs (and computer graphics) of regular wave patterns in a laboratory. The experiment was designed to verify that wave patterns of a genus 2 soliton interaction of the KP (Kadomtsev-Petviashvili) equation could actually be produced, giving practical confirmation of the validity of the KP equation to model the interaction of long waves of small but finite amplitude that travel in slightly different directions. Assuming the KP, or any other equation, to be an accurate model, 
the question arises whether a unique solution can be found that matches the geometry of observed wave patterns, so without information of the actual wave heights. If so, then this solution would produce the wave heights from a photographic picture of the wave pattern, which would be useful for hydrodynamic laboratories where often only (a very limited number of) point measurements are available for rather complicated multi-directional wave patterns that arise, for instance, from diffraction at a ship. In this and a subsequent paper we will study this inverse problem: from the geometry of the wave pattern to a specific solution of the model equation.

In order to tackle the inverse problem, first the direct problem has to be solved, which means to find all the possible wave patterns of the solutions of the model equation. Even when the most simple situation is considered (no winds, no currents, incompressible fluid without surface tension, etc.), the free surface equations are well known but too complicated for a direct investigation of the wave patterns. Therefore, we will take an alternative approach, supported by the observation that "soliton" interactions seem to be rather robust phenomena in (the theory of) water waves. Hence, as a first step, we will associate wave crests with solitons. There are various possibilities to choose from equations that produce solitons; keeping some flexibility, we shall consider the direct and inverse problem for the large class of so-called KdV (Korteweg-de Vries) type of equations. This is a well defined class of equations in one or two (or more) space variables for which solitons and their interactions can be studied analytically; this class has been identified by Grammaticos et al. [3].

For the study of soliton interactions, Hirota's formalism [4] turns out to be most suited and produces well-structured expressions for soliton solutions of arbitrary genus $g$ (the number of pure solitons). The detailed analysis is carried out in this paper in two steps along the following lines. First we describe wave patterns in a precise and algorithmic way in the ( $g$ dimensional) space of phase variables. Then a mapping to real spatial-temporal variables is performed to obtain an observable evolution of the free surface. In order to restrict the analysis to essentials, we investigate in this paper solitons of genus 2 , leaving the general case to a next paper. However, we use a self-contained simple notation that supports the understanding of the general expressions for genus $g$.

As an important aspect of our analysis, we will describe a two-soliton solution as a sum $U=S_{1}+S_{2}+S_{12}$, in which $S_{1}, S_{2}$ represent two pure solitons, while $S_{12}$ will be an interaction soliton; this interaction soliton plays a fundamental role in analyzing the interaction. It should be observed that this decomposition is different from the "eigenvalue and eigenfunction decomposition" introduced by Gardner et al. [5] (GGKM) in the context of the inverse scattering theory. (See also Yoneyama [6] for the interpretation, Hodnett and Moloney [7] for the equivalent representation of the GGKM decomposition). The proposed decomposition is also different from the "decomposition of the multi-soliton 
as a linear superposition of accelerating solitary waves and interaction terms", as proposed by Bryan and Stuart [8]: in our case, the number of terms is $2^{g}-1$ instead of $2 g$, and there is no need for the notion of acceleration.

A final remark concerns many of the studies on the interaction of solitons that are modelled by the classical KdV equation. This model, however, has significant limitations. For example, Hirota and Ito [9] have pointed out that in the case of the Sawada-Kotera equation, the interaction of two 1D solitons emits and absorbs, under certain conditions, a third — interaction — soliton. We found that this is actually a universal phenomenon, especially when solitons in higher dimensions than one are considered. The reason that one cannot observe this phenomenon in the classical KdV case can be understood by realising that, in order to reveal the third soliton, the phase shift of the two interacting solitons should be rather large, which would imply the KdV solitons to be nearly equal. In the study of LeVeque [10] devoted to this phenomenon, for example, the third soliton is not recognized because its amplitude vanishes when the phase shift is increased.

The organization of this paper is as follows. In Section 2 we briefly recall some results about the KdV-type equations and Hirota's bilinear formalism, and then present in Section 3 the notation and some auxiliary results that will simplify the analysis. In Section 4 we analyze the two-soliton solution in phase variables where the new perspectives to understand soliton interactions are introduced: the concept of the interaction soliton and the specific decomposition of the two-soliton solution in Section 4.1, and a geometric representation of the soliton interaction in Section 4.2. Then, in Section 5, the description in phase variables is transformed to real spatial-temporal variables. The actual inverse problem is solved in Section 6 where an explicit solution algorithm is given for solving the KP equation.

\section{KdV-type equations and Hirota's bilinear formalism}

Let us consider a nonlinear partial differential equation

$$
K\left(u, u_{x}, u_{y}, u_{t}, \ldots\right)=0
$$

where subscripts denote partial derivation of the function $u=u(x, y, t)$, and introduce a change of dependent variables, say [3]

$$
u=2 \frac{\partial^{2}}{\partial x^{2}} \ln \theta
$$


which results in an equation for the function $\theta=\theta(x, y, t)$ :

$$
G\left(\theta, \theta_{x}, \theta_{y}, \theta_{t}, \ldots\right)=0
$$

We have to specify $\mathrm{KdV}$-type equations in more detail compared with [3, 11]. Here follows our definition for the KdV-type equations:

Definition 1 Equation (1) is said to be a KdV-type equation iff there exists a polynomial (Hirota polynomial) $P=P(\mu, \nu, \omega)$ with the following properties:

(1) $P(0,0,0)=0, P(-\mu,-\nu,-\omega)=P(\mu, \nu, \omega)$;

(2) all nontrivial solutions of Hirota bilinear form

$$
P\left(D_{x}, D_{y}, D_{t}\right) \theta \cdot \theta=0
$$

are the solutions to Eq. (3) (and then, through (2), also the solutions to (1)).

Here symbols $D_{x}, D_{y}, D_{t}$ denote Hirota derivatives, acting on a pair of functions $(f, g)$ as follows (we denote $\boldsymbol{x}=(x, y)^{\mathrm{T}}$ )

$$
\begin{aligned}
\left(D_{x}^{n} D_{y}^{m} D_{t}^{k} f \cdot g\right) & (\boldsymbol{x}, t) \equiv \\
& \left.\left(\partial_{x}-\partial_{x^{\prime}}\right)^{n}\left(\partial_{y}-\partial_{y^{\prime}}\right)^{m}\left(\partial_{t}-\partial_{t^{\prime}}\right)^{k} f(\boldsymbol{x}, t) g\left(\boldsymbol{x}^{\prime}, t^{\prime}\right)\right|_{\left(\boldsymbol{x}^{\prime}, t^{\prime}\right)=(\boldsymbol{x}, t)}
\end{aligned}
$$

In this paper we illustrate our theory using the KP equation in normalized variables [12]:

$$
\left(u_{t}+6 u u_{x}+u_{x x x}\right)_{x}+3 u_{y y}=0
$$

The KP equation is KdV-type equation with the change of variables (2) and the corresponding Hirota polynomial is $P(\mu, \nu, \omega)=\mu \omega+\mu^{4}+3 \nu^{2}$.

When the Hirota bilinear form (4) is found for the initial equation (1), special solutions like soliton solutions can be easily obtained from the observation that the eigenproblem of Hirota's operator $\tilde{P}=P\left(D_{x}, D_{y}, D_{t}\right)$, that is,

$$
\tilde{P} f \cdot g=\lambda f g
$$

has the following solution

$$
\begin{aligned}
(f, g) & =\left(e^{\mu x+\nu y+\omega t}, e^{\mu^{\prime} x+\nu^{\prime} y+\omega^{\prime} t}\right), \\
\lambda & =P\left(\mu-\mu^{\prime}, \nu-\nu^{\prime}, \omega-\omega^{\prime}\right),
\end{aligned}
$$

where $\mu, \mu^{\prime}, \ldots \in \mathbb{R}$. So, if the solution of (4) is looked at as a certain polynomial of exponential functions then by using (6) and (7), one obtains a system 
of algebraic equations for the parameters $\mu, \nu$, etc. For example, a two-soliton solution is found to be

$$
\theta=1+e^{\mu_{1} x+\nu_{1} y+\omega_{1} t}+e^{\mu_{2} x+\nu_{2} y+\omega_{2} t}+A_{12} e^{\mu_{1} x+\nu_{1} y+\omega_{1} t+\mu_{2} x+\nu_{2} y+\omega_{2} t},
$$

where the parameters $\mu_{i}, \nu_{i}, \omega_{i}, i=1,2$, must satisfy the dispersion relations $P\left(\mu_{i}, \nu_{i}, \omega_{i}\right)=0$; and the coefficient $A_{12}$ is found as a solution of the algebraic equations:

$$
A_{12}=-\frac{P\left(\mu_{1}-\mu_{2}, \nu_{1}-\nu_{2}, \omega_{1}-\omega_{2}\right)}{P\left(\mu_{1}+\mu_{2}, \nu_{1}+\nu_{2}, \omega_{1}+\omega_{2}\right)} .
$$

Remark: When the genus (number of solitons) is larger than two, there will be additional (Hirota) conditions which will be dealt with in a successive paper.

\section{$3 \quad$ Notation and auxiliary results}

The core of this study is formed by the analysis of the functional form (2) with a theta-function generating soliton solutions. In this section we introduce appropriate notation and present some auxiliary results needed for the consistent analysis.

First, $\boldsymbol{x}=(x, y)^{\mathrm{T}}$ and $\boldsymbol{\varphi}=\left(\varphi_{1}, \ldots, \varphi_{g}\right)^{\mathrm{T}}$ denote 2-vector and $g$-vector of real space and phase variables, respectively. Functions defined on the space of real spatial-temporal variables and of phase variables are denoted by lower and upper case symbols, respectively: $\theta=\theta(\boldsymbol{x}, t), u=u(\boldsymbol{x}, t)$ and $\Theta=\Theta(\boldsymbol{\varphi})$, $U=U(\boldsymbol{\varphi})$, for example.

Second, real and phase variables are related:

$$
\boldsymbol{\varphi}(\boldsymbol{x}, t)=\boldsymbol{\mu} x+\boldsymbol{\nu} y+\boldsymbol{\omega} t+\boldsymbol{\varphi}_{0}
$$

where $\boldsymbol{\mu}, \boldsymbol{\nu}, \boldsymbol{\omega}, \boldsymbol{\varphi}_{0}$ are $g$-vectors. We also write $\boldsymbol{\varphi}=\mathbf{K} \boldsymbol{x}+\boldsymbol{\omega} t+\boldsymbol{\varphi}_{0}$, where wave matrix $\mathbf{K}$ contains the vectors $\boldsymbol{\mu}, \boldsymbol{\nu}$ as column vectors. The row vectors of $\mathbf{K}$ are wave vectors $\boldsymbol{k}_{i}^{\mathrm{T}}=\left(\mu_{i}, \nu_{i}\right)$ so that we have $\varphi_{i}=\boldsymbol{k}_{i}^{\mathrm{T}} \boldsymbol{x}+\omega_{i} t+\varphi_{0 i}, i=1, \ldots, g$.

Third, the functions of real and phase variables are related: $\theta(\boldsymbol{x}, t)=\Theta(\boldsymbol{\varphi}(\boldsymbol{x}, t))$, $u(\boldsymbol{x}, t)=U(\boldsymbol{\varphi}(\boldsymbol{x}, t))$.

Fourth, for functions (denoted by $U$ and only) indices are used to indicate the number of arguments. For example, $U_{n}$ is a function of $n$ variables. If $n=g$, this index is not shown so that $U(\boldsymbol{\varphi}) \equiv U_{g}\left(\varphi_{1}, \ldots, \varphi_{g}\right)$. The reason for this convention is that in the following $U$ represents a soliton solution and the 
index determines its genus, especially if the soliton solution is obtained as a result of some limiting process on the soliton solution of higher genus, say $g$.

Finally, the change of variables (2) motivates to introduce an operator $L$ :

$$
L=2\left(\mu_{1} \frac{\partial}{\partial \varphi_{1}}+\ldots+\mu_{g} \frac{\partial}{\partial \varphi_{g}}\right)^{2} \ln .
$$

Then $U(\boldsymbol{\varphi})=L[\Theta(\boldsymbol{\varphi})]$ and the following properties hold:

(1) $L$ is gauge invariant (follows from (13) and $L\left[e^{r^{\mathrm{T}} \boldsymbol{\varphi}}\right]=0$ ):

$$
L\left[e^{\boldsymbol{r}^{\mathrm{T}} \boldsymbol{\varphi}} \Theta\right]=L[\Theta], \quad \forall \boldsymbol{r} \in \mathbb{R}^{g} .
$$

If $\Theta^{\prime}=e^{r^{\mathrm{T}} \boldsymbol{\varphi}} \Theta$ then we say that theta-functions $\Theta^{\prime}$ and $\Theta$ are equivalent.

(2) Operators $L$ and $\lim _{\varphi_{i} \rightarrow \pm \infty}$ commute in the space of all finite polynomials of exponential functions (see Appendix A):

$$
\lim _{\varphi_{i} \rightarrow \pm \infty} L[\Theta(\boldsymbol{\varphi})]=L\left[\lim _{\varphi_{i} \rightarrow \pm \infty} \Theta(\boldsymbol{\varphi})\right]
$$

if $\Theta(\boldsymbol{\varphi})=\sum_{\boldsymbol{m} \in I \subset \mathbb{Z}^{g}} A_{\boldsymbol{m}} e^{\boldsymbol{m}^{\mathrm{T}} \boldsymbol{\varphi}}$ and $|I|<\infty$. Note that (12) makes sense only if $\lim _{\varphi_{i} \rightarrow \pm \infty} \Theta(\boldsymbol{\varphi})$ exists and is nonzero. Otherwise, one should first apply the gauge invariance of $L$ before using the formula.

(3) We have

$$
L\left[\Theta_{1} \Theta_{2}\right]=L\left[\Theta_{1}\right]+L\left[\Theta_{2}\right]
$$

(4) If $\Theta=1+e^{\varphi_{i}}$ then we have an one-soliton solution in phase variables:

$$
U_{1}\left(\varphi_{i}\right)=L\left[1+e^{\varphi_{i}}\right]=\frac{\mu_{i}^{2}}{2} \operatorname{sech}^{2} \frac{\varphi_{i}}{2} .
$$

In general, the function $\Theta=\Theta(\varphi)$ is a certain polynomial of exponential functions $e^{m^{\mathrm{T}} \boldsymbol{\varphi}}$ that is found by solving the corresponding bilinear form (4) of Eq. (1) (see Section 2).

\section{Two-soliton solution in phase variables}

In this Section we present a detailed study of the two-soliton solution in phase variables, which is written in the form $U\left(\varphi_{1}, \varphi_{2}\right)=L\left[\Theta\left(\varphi_{1}, \varphi_{2}\right)\right]$, where $\Theta$ is the following theta-function:

$$
\Theta\left(\varphi_{1}, \varphi_{2}\right)=1+e^{\varphi_{1}}+e^{\varphi_{2}}+A_{12} e^{\varphi_{1}+\varphi_{2}}
$$

We assume $A_{12} \geqslant 0$ because we are not interested in singular cases. 


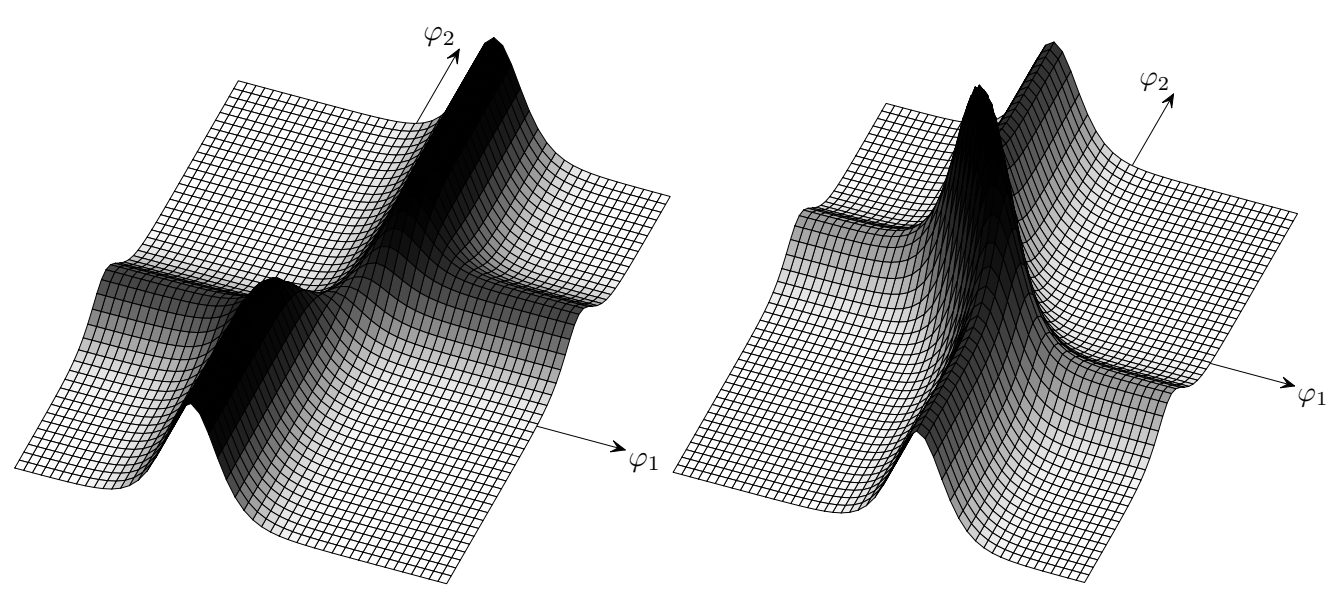

Fig. 2. Two-soliton solutions with positive $\left(0<A_{12}<1\right.$, left plot $)$ and negative $\left(A_{12}>1\right.$, right plot $)$ phase shifts.

The behavior of the two-soliton solution as the phase variables $\varphi_{1}, \varphi_{2}$ are changed from $-\infty$ to $+\infty$ is described by the following equations (see Appendix B, (B.1) and (B.2))

$$
\begin{aligned}
\lim _{\varphi_{1} \rightarrow-\infty} U\left(\varphi_{1}, \varphi_{2}\right) & =U_{1}\left(\varphi_{2}\right), & \lim _{\varphi_{1} \rightarrow+\infty} U\left(\varphi_{1}, \varphi_{2}\right) & =U_{1}\left(\varphi_{2}+\ln A_{12}\right), \\
\lim _{\varphi_{2} \rightarrow-\infty} U\left(\varphi_{1}, \varphi_{2}\right) & =U_{1}\left(\varphi_{1}\right), & \lim _{\varphi_{2} \rightarrow+\infty} U\left(\varphi_{1}, \varphi_{2}\right) & =U_{1}\left(\varphi_{1}+\ln A_{12}\right),
\end{aligned}
$$

that is, in these limit processes the two solitons (represented here by $U_{1}$ ) undergo a phase shift $\Delta_{12}=-\ln A_{12}$. Note that if $A_{12}<1$, the value of the phase shift $\Delta_{12}$ is positive while for $A_{12}>1$ it is negative. The amplitudes of the two solitons are $\frac{1}{2} \mu_{1}^{2}$ and $\frac{1}{2} \mu_{2}^{2}$, respectively. Figure 2 illustrates the function $U\left(\varphi_{1}, \varphi_{2}\right)$ with positive and negative phase shift, respectively.

For different values of the coefficient $A_{12}$ two special cases can be distinguished.

Case $A_{12}=0$ : The two-soliton solution represents a resonance of two solitons. Here, different from (16) and (17), the limit processes $\varphi_{1}, \varphi_{2} \rightarrow+\infty$ give zeros. But the limit process $\varphi_{1}+\varphi_{2} \rightarrow+\infty$ with $\varphi_{1}-\varphi_{2}$ constant, gives an one-soliton solution (see (B.3)):

$$
\lim _{\substack{\varphi_{1}+\varphi_{2} \rightarrow+\infty \\\left|\varphi_{1}-\varphi_{2}\right|<\infty}} U\left(\varphi_{1}, \varphi_{2}\right)=U_{1}\left(\varphi_{1}-\varphi_{2}\right)
$$

The corresponding soliton is called a resonance soliton. Note that from $A_{12}=0$ (see (8)) the dispersion relation $P\left(\mu_{1}-\mu_{2}, \nu_{1}-\nu_{2}, \omega_{1}-\omega_{2}\right)=0$ follows for the resonance soliton. So, the use of notion "soliton" is justified. The amplitude of the resonance soliton is $\frac{1}{2}\left(\mu_{1}-\mu_{2}\right)^{2}$. Figure 3 illustrates the two-soliton solution in phase variables with the resonance soliton. This case can be interpreted as a two-soliton solution with an infinite phase shift: $\Delta_{12}=+\infty$. 


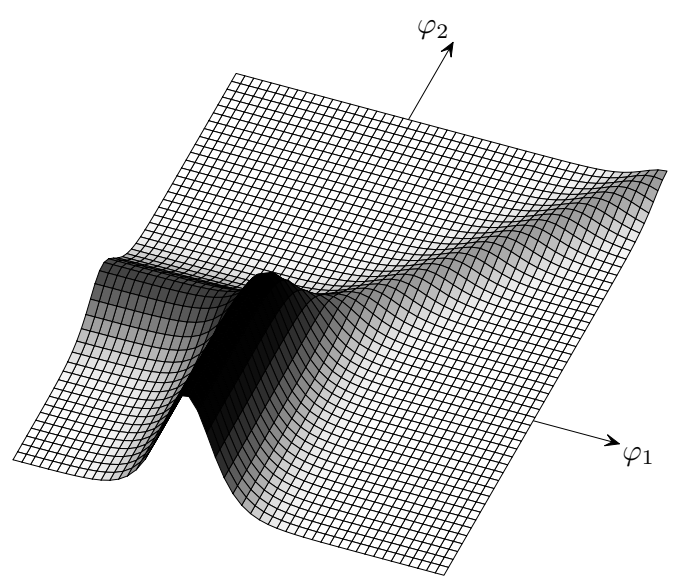

Fig. 3. From the interaction of solitons a resonance soliton may be formed.
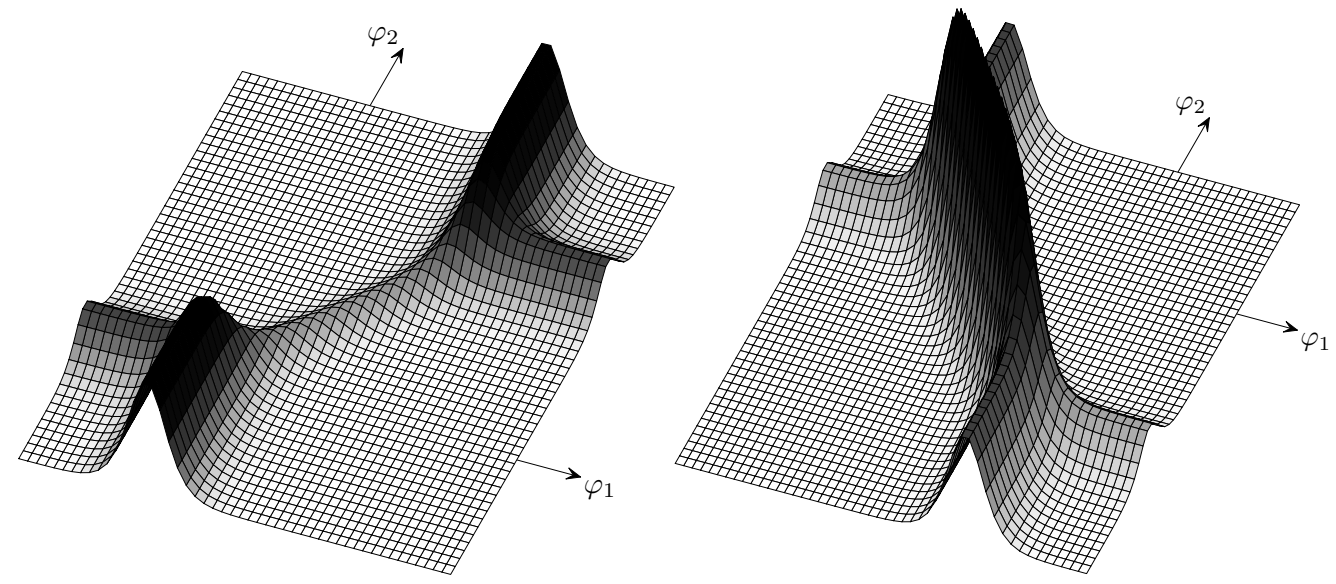

Fig. 4. Two-soliton solutions with large positive (left plot) and large negative (right plot) phase shifts. The interaction solitons are well visible.

Case $A_{12}=+\infty$ The two-soliton solution is trivial. That is, we have (see (B.4))

$$
\lim _{A_{12} \rightarrow+\infty} U\left(\varphi_{1}, \varphi_{2}\right)=0 .
$$

This result can be interpreted as follows. During the given limit process the phase shift is negative and approaches minus infinity: $\Delta_{12} \rightarrow-\infty$. As a result, the solitons move away from our field of vision, if we sit at the origin of the space of phase variables (c.f. Fig. 4, right plot).

\subsection{Interaction soliton and the decomposition of a two-soliton solution}

The structure of the two soliton interaction clears up when we consider cases near to the limiting cases $A_{12}=0$ and $A_{12}=+\infty$. Figure 4 contains plots of the two-soliton solutions corresponding to the cases $0<A_{12} \ll 1$ and $A_{12} \gg 1$. These plots show that during the interaction a soliton-like profile forms that we call an interaction soliton. 

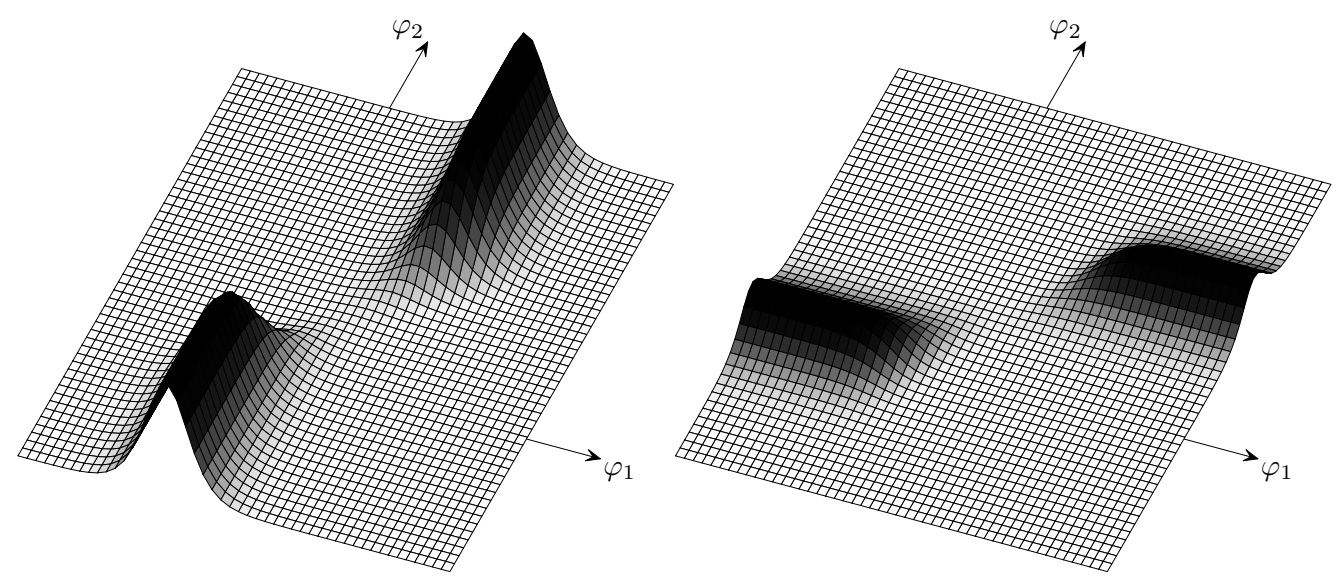

Fig. 5. Functions $S_{1}\left(\varphi_{1}, \varphi_{2}\right)$ and $S_{2}\left(\varphi_{1}, \varphi_{2}\right)$ corresponding to the first and second pure soliton, respectively.

Below we give a more precise definition of the interaction soliton. For that we introduce the following theta-function

$$
\Theta^{\prime}\left(\varphi_{1}, \varphi_{2}\right)=\cosh \frac{\varphi_{1}-\varphi_{2}}{2}+\sqrt{A_{12}} \cosh \frac{\varphi_{1}+\varphi_{2}+\ln A_{12}}{2}
$$

that is equivalent with (15) (see (B.5)). Using this $\Theta^{\prime}$, one can derive the following expression for the two-soliton solution in phase variables:

$$
\begin{array}{r}
U\left(\varphi_{1}, \varphi_{2}\right)=\left(\sqrt{A_{12}}\left(\mu_{1}^{2} \cosh \left(\varphi_{2}+\ln \sqrt{A_{12}}\right)+\mu_{2}^{2} \cosh \left(\varphi_{1}+\ln \sqrt{A_{12}}\right)\right)\right. \\
\left.+\frac{\left(\mu_{1}-\mu_{2}\right)^{2}+A_{12}\left(\mu_{1}+\mu_{2}\right)^{2}}{2}\right) \frac{1}{\Theta^{\prime 2}}
\end{array}
$$

This suggests the following decomposition:

$$
U\left(\varphi_{1}, \varphi_{2}\right)=S_{1}\left(\varphi_{1}, \varphi_{2}\right)+S_{2}\left(\varphi_{1}, \varphi_{2}\right)+S_{12}\left(\varphi_{1}, \varphi_{2}\right)
$$

where the constituent functions are given by

$$
\begin{aligned}
S_{1}\left(\varphi_{1}, \varphi_{2}\right) & =\frac{\sqrt{A_{12}} \mu_{1}^{2} \cosh \left(\varphi_{2}+\ln \sqrt{A_{12}}\right)}{\Theta^{\prime 2}}, \\
S_{2}\left(\varphi_{1}, \varphi_{2}\right) & =\frac{\sqrt{A_{12}} \mu_{2}^{2} \cosh \left(\varphi_{1}+\ln \sqrt{A_{12}}\right)}{\Theta^{\prime 2}} \\
S_{12}\left(\varphi_{1}, \varphi_{2}\right) & =\frac{\left(\mu_{1}-\mu_{2}\right)^{2}+A_{12}\left(\mu_{1}+\mu_{2}\right)^{2}}{2 \Theta^{\prime 2}} .
\end{aligned}
$$

The plots of these functions (with $A_{12}<1$ ) are shown in Figs. 5 and 6; so that $S_{1}, S_{2}$, and $S_{12}$ correspond to two pure solitons and the interaction soliton, 

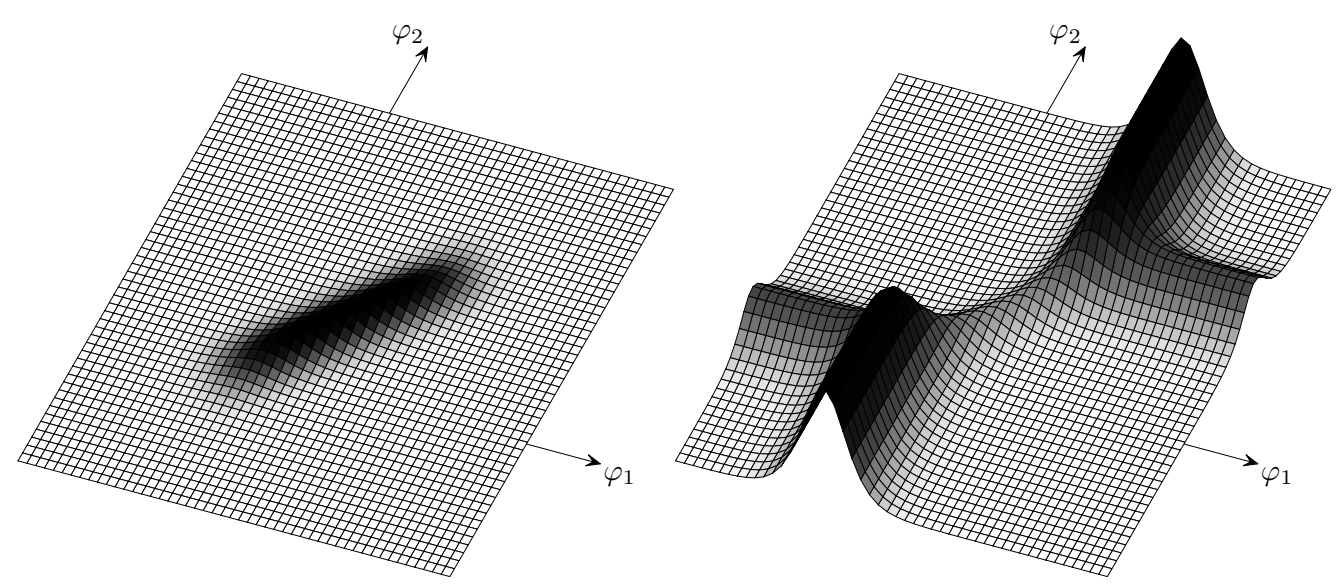

Fig. 6. Left plot: A function $S_{12}\left(\varphi_{1}, \varphi_{2}\right)$ corresponding to the interaction soliton. Right plot: Linear superposition of the two pure solitons $S_{1}, S_{2}$ and the interaction soliton $S_{12}$ gives the two-soliton solution.

respectively. They also satisfy the following properties:

$$
\begin{aligned}
\lim _{\varphi_{2} \rightarrow-\infty} S_{1}\left(\varphi_{1}, \varphi_{2}\right) & =U_{1}\left(\varphi_{1}\right), & \lim _{\varphi_{2} \rightarrow+\infty} S_{1}\left(\varphi_{1}, \varphi_{2}\right) & =U_{1}\left(\varphi_{1}+\ln A_{12}\right), \\
\lim _{\varphi_{1} \rightarrow-\infty} S_{2}\left(\varphi_{1}, \varphi_{2}\right) & =U_{1}\left(\varphi_{2}\right), & \lim _{\varphi_{1} \rightarrow+\infty} S_{2}\left(\varphi_{1}, \varphi_{2}\right) & =U_{1}\left(\varphi_{2}+\ln A_{12}\right), \\
\lim _{\varphi_{i} \rightarrow \pm \infty} S_{i}\left(\varphi_{1}, \varphi_{2}\right) & =0, & \lim _{\varphi_{i} \rightarrow \pm \infty} S_{12}\left(\varphi_{1}, \varphi_{2}\right) & =0,
\end{aligned}
$$

where $i=1,2$, which justify stating that the two-soliton solution $U$ is a linear superposition of the two pure solitons $S_{1}, S_{2}$ and the interaction soliton $S_{12}$.

The amplitude of the interaction soliton is defined as a value of the function $S_{12}$ at the center of the interaction region: $S_{12}\left(\frac{1}{2} \Delta_{12}, \frac{1}{2} \Delta_{12}\right)$. It depends on the coefficient $A_{12}$ as follows: if $A_{12}$ is changed from zero to $+\infty$, then the amplitude of the interaction soliton varies monotonically from $\frac{1}{2}\left(\mu_{1}-\mu_{2}\right)^{2}$ to $\frac{1}{2}\left(\mu_{1}+\mu_{2}\right)^{2}$.

As for example, Figure 7 illustrates how the coefficient $A_{12}$ depends on the wave parameters $\mu_{1}>0, \mu_{2}>0, \nu_{1}, \nu_{2}$ in the KP equation case. For nonzero interaction angle (represented by $\rho$ ), the interactions of two KP solitons may have positive and negative phase shifts (if $A_{12}>1$ and $A_{12}<1$, respectively). Resonance soliton forms on the line denoted by $A_{12}=0$. The interaction is singular in regions with $A_{12}<0$. In regions, where $A_{12}$ is close to zero or very large, the interaction soliton is well visible.

\subsection{Geometric representation of soliton interaction}

We introduce a simplified description for the interaction process of two solitons. Namely, we describe the geometry of the interaction picture by the two 


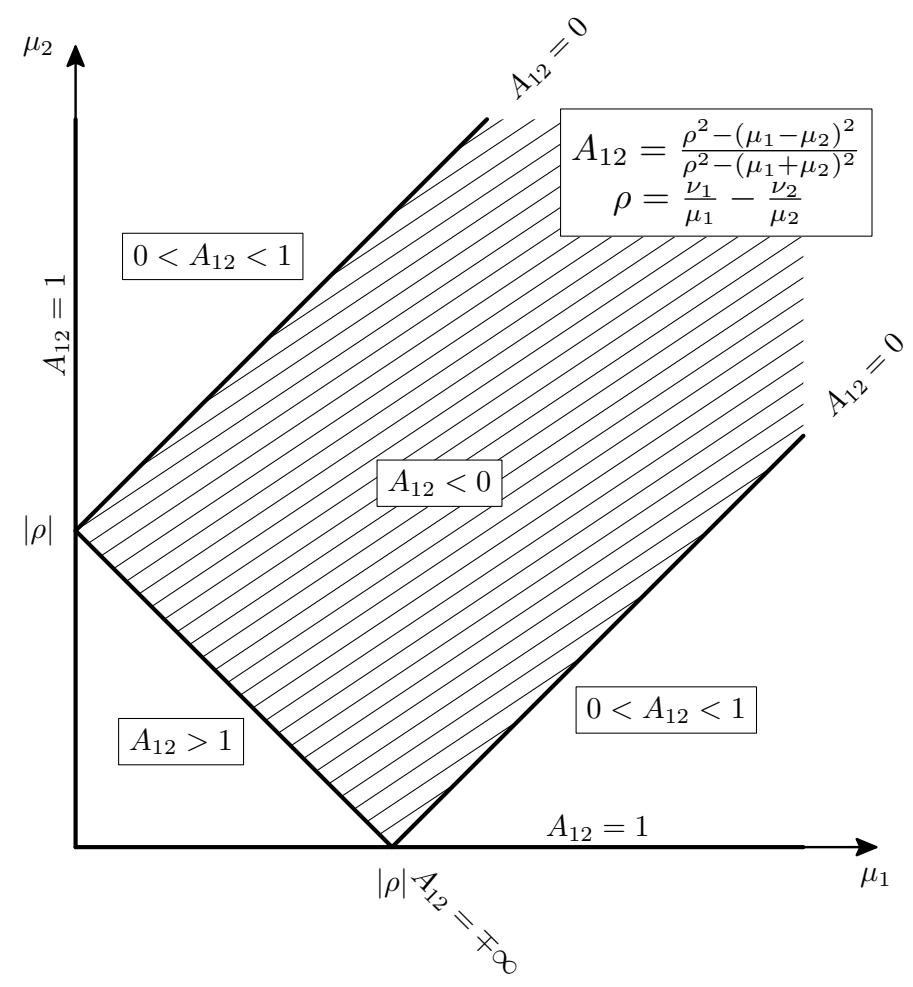

Fig. 7. "Map" of soliton types for the KP equation.
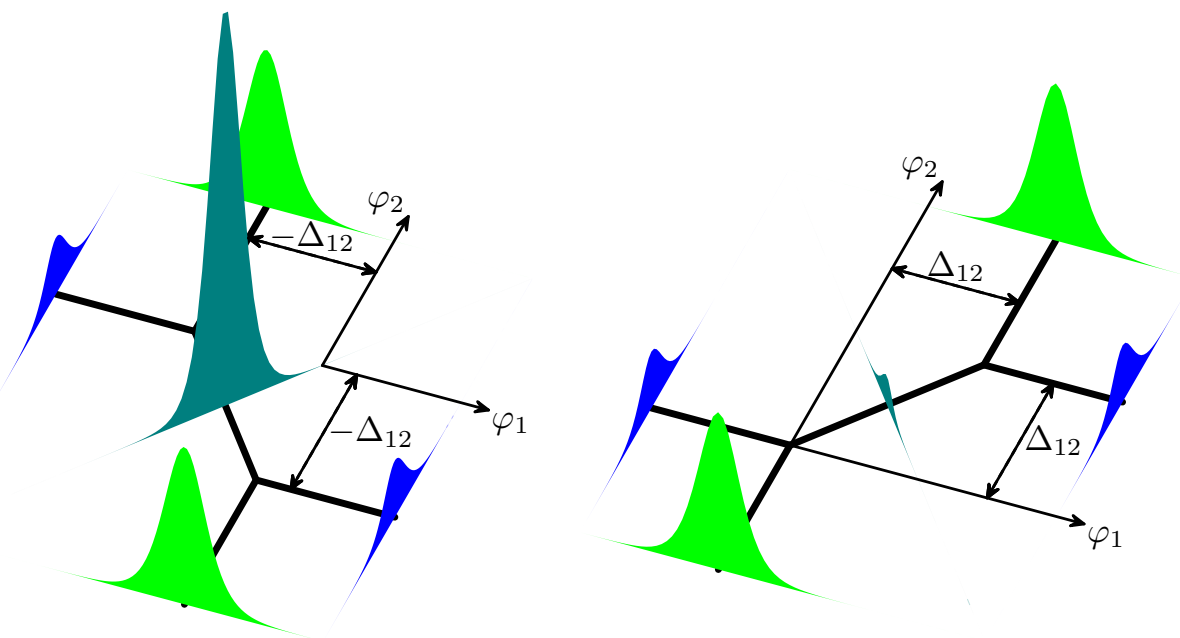

Fig. 8. Phase patterns (bold lines) of the two-soliton solution with negative and positive phase shift, respectively.

pairs of parallel line slices connected with a diagonal line slice as shown in Fig. 8. One set of parallel line slices describes the position of one soliton and its shifted counterpart in an approximate way; and the diagonal line slice shows the position of the interaction soliton. We call the whole set of these line elements a phase pattern set and denote it by $P_{U}$.

Note that the phase pattern set $P_{U}$ of the two-soliton solution is consistent 
with the decomposition introduced in Section 4.1. The two pairs of parallel line slices show approximately the positions of the "crests" of the pure solitons $S_{1}$ and $S_{2}$, respectively. The diagonal line slice corresponds to the interaction term $S_{12}$ in the same sense.

\section{Two-soliton solution in real variables}

In this Section we complete the direct problem by transferring the results obtained for the two-soliton solution in phase variables to the ones in real spatial-temporal variables. For that we use the relation $u(\boldsymbol{x}, t)=U(\boldsymbol{\varphi}(\boldsymbol{x}, t))$ and the particular form of the mapping $\boldsymbol{\varphi}(\boldsymbol{x}, t)$ given in $(9)$.

With the phase pattern set $P_{U}$ of the two-soliton solution $U=U(\boldsymbol{\varphi})$ (see Section 4.2) the corresponding function $u=u(\boldsymbol{x}, t)$ has a real pattern set $P_{u}(t)$, defined by

$$
P_{u}(t)=\left\{\boldsymbol{x} \mid \mathbf{K} \boldsymbol{x}+\boldsymbol{\omega} t+\boldsymbol{\varphi}_{0} \in P_{U}\right\}
$$

The direct problem is solved if we manage to construct the real pattern set $P_{u}(t)$ for the function $u=u(\boldsymbol{x}, t)$ for any time moment. In the general case this is not trivial since then the dimensions of the spaces of real spatial and phase variables (the dimensions are 2 and $g$, respectively) can be different, especially for $g$ being large.

In the following we assume $g=2$ that corresponds to the evolution of two solitons in two spatial dimensions. Without loss of generality, we also assume $\boldsymbol{\varphi}_{0}=\mathbf{0}$ and that the wave vectors $\boldsymbol{k}_{1}, \boldsymbol{k}_{2}$ are not collinear.

With these assumptions the wave matrix $\mathbf{K}$ defines an affine mapping between the two-dimensional planes of real and phase variables: $\boldsymbol{\varphi}=\mathbf{K} \boldsymbol{x}$. The definition of the real pattern set (19) is simplified to the following relation

$$
P_{U}=\mathbf{K} P_{u}(t)+\boldsymbol{\omega} t
$$

Since the mapping $\mathbf{K}: \mathbb{R}^{2} \rightarrow \mathbb{R}^{2}$ is also bijective, we can write the expression for the real pattern $P_{u}(t)$ explicitly as follows

$$
P_{u}(t)=\mathbf{K}^{-1} P_{U}-\boldsymbol{w} t
$$

where $\boldsymbol{w}=\mathbf{K}^{-1} \boldsymbol{\omega}$

The first term in the formula (20) gives the real pattern at time $t=0$. For increasing time, this pattern is just shifted in the opposite direction of the 

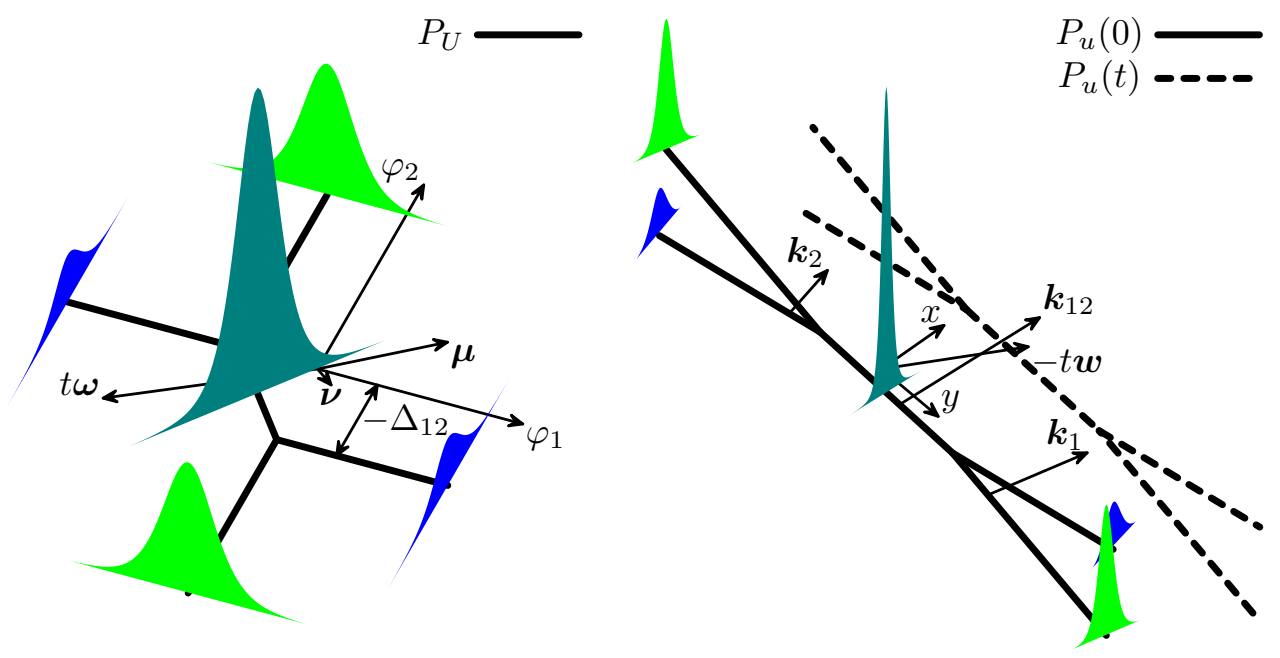

Fig. 9. The negative phase shift case: The geometric representation of the two-soliton solution in phase variables (left) and the dynamics of the two-soliton pattern in the real space (right).
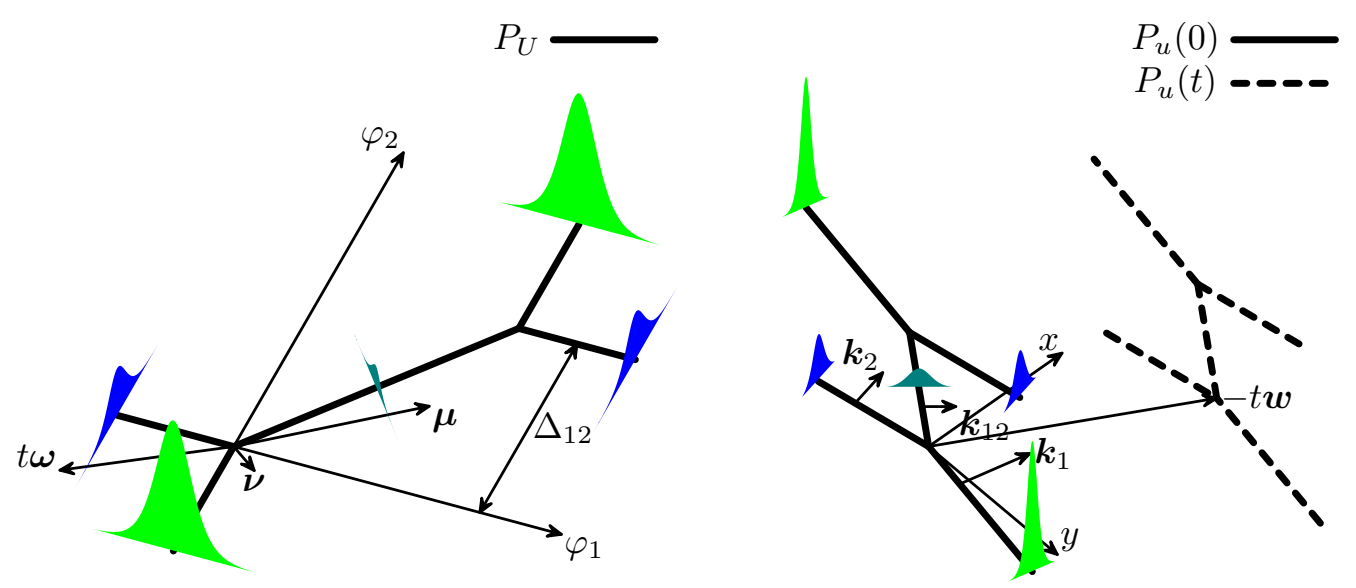

Fig. 10. The positive phase shift case (see Fig. 9).

vector $\boldsymbol{w}$ : in the frame of reference moving with velocity vector $\boldsymbol{w}$, the pattern of the two-soliton solution is stationary.

Figures 9 and 10 illustrate the relation between the phase and real space variables and the dynamics of the real pattern of the two-soliton solution. Observe that the vectors $\boldsymbol{\mu}$ and $\boldsymbol{\nu}$ are the images of the coordinate vectors in the real space. If time increases, these vectors are shifted in the direction of the vector $\boldsymbol{\omega}$ (in Figs. 9 and 10 the vectors $\boldsymbol{\mu}, \boldsymbol{\nu}$ are plotted for time $t=0$ ). 

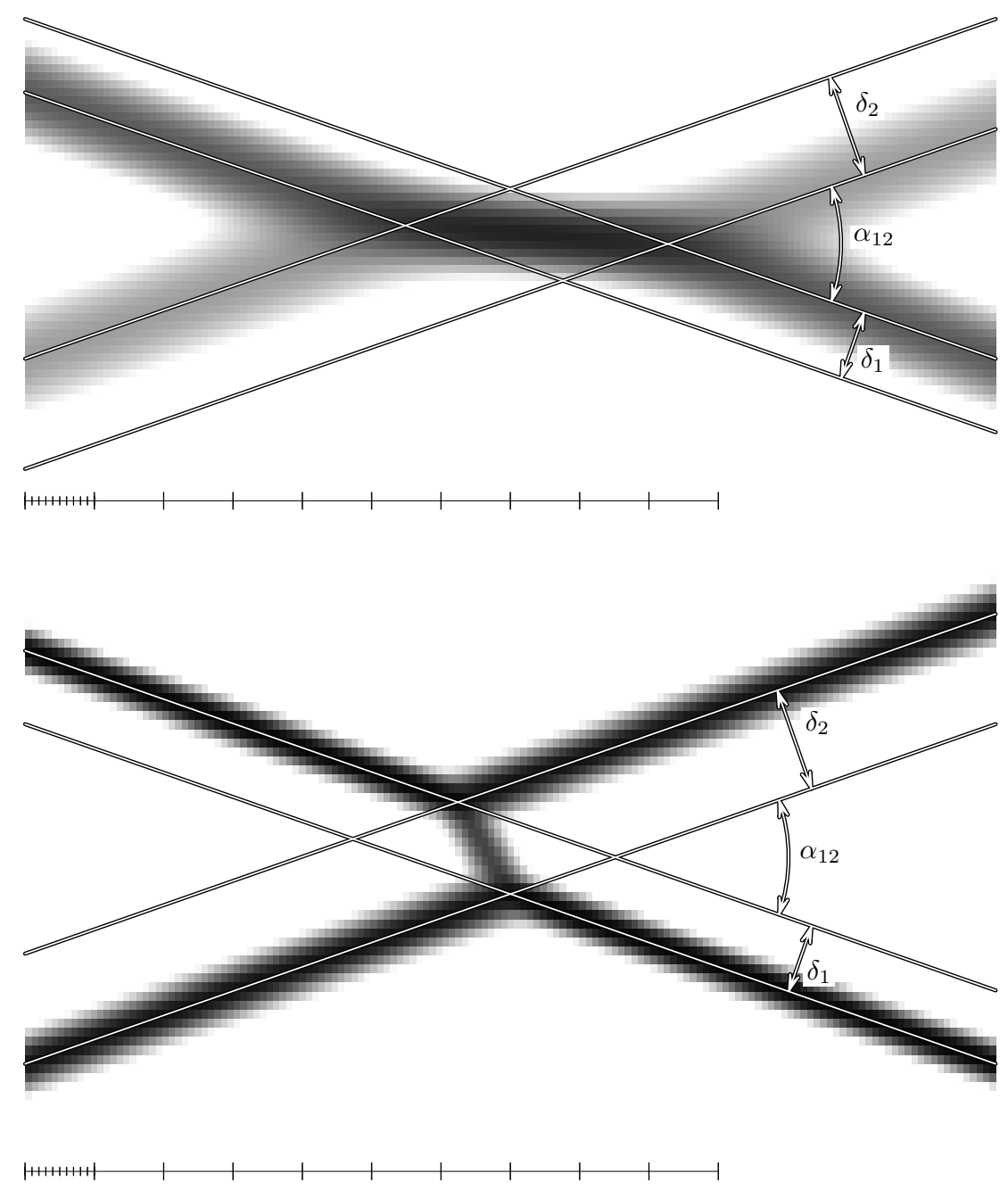

Fig. 11. Interaction patterns of the two-soliton solution corresponding to the negative (upper figure) and positive (lower figure) phase shift cases.

\section{Inverse problem}

The starting point for this Section is a wave pattern picture. Our aim is to determine the parameters (most important, the amplitudes) of the waves seen in this pattern picture. In this paper we limit our study to two waves in two dimensions. These cases are modeled by two-soliton interactions. Below we first overview the main steps in solving the inverse problem and then illustrate the solution for the KP model.

Recall from Section 5 that the configuration of the wave pattern of the twosoliton solution in phase variables is determined by one phase shift parameter $\Delta_{12}$ that can have either negative or positive values, in general. Figure 11 
shows that the configuration of the corresponding wave pattern in the real space is determined by three parameters. These are two shift parameters $\delta_{1}, \delta_{2}$ that measure the shifts of the wave crests resulted from the interaction process, and one parameter $\alpha_{12}$ measuring the relative angle between the wave crests. So, three equations can be stated for relating these measured parameters with wave parameters.

On the other hand, the two-soliton solution is determined by four parameters: $\mu_{1}, \mu_{2}, \nu_{1}, \nu_{2}$. Consequently, at this point one equation is absent in order to obtain a complete system of equations for four unknowns. This equation results by specifying a concrete physical model, which is done below.

In general, solving the inverse problem of wave patterns modeled by multisoliton solution, consists of the following four main steps:

(1) Measure the shifts of the wave crests and state the equations relating these measurements with the parameters in the wave vectors.

(2) Measure the relative angles between the wave crests and state the corresponding equations.

(3) Apply the assumptions of the physical model at hand in order to obtain a complete set of equations.

(4) Finally, solve the system of equations. In the case of multiple solutions, choose the ones that make sense physically.

Note that in finding the equations for steps 1 and 2, where only distances and angles are of importance, we can use a simplified version of equation (9): $\boldsymbol{\varphi}=\mathbf{K} \boldsymbol{x}+\boldsymbol{\varphi}_{0}$. Moreover, since we only consider the case $g=2$, we can take $\varphi_{0}=0$.

In the following we derive the system of equations of the inverse problem for two KP waves.

Relations for the shifts of wave crests: Figure 12 illustrates how the wave patterns with negative and positive phase shift (left figures) are mapped with $\boldsymbol{\varphi}=\mathbf{K} \boldsymbol{x}$ to the space of phase variables (right figures). In particular, vectors $\boldsymbol{\delta}_{1}, \boldsymbol{\delta}_{2}$, that characterize the shifts undergone by the two waves, are mapped to vectors $\mathbf{K} \boldsymbol{\delta}_{i}, i=1,2$, in the space of phase variables. Note that one of the coordinates of the both vectors $\mathbf{K} \boldsymbol{\delta}_{i}, i=1,2$, must be equal to $-\left|\Delta_{12}\right|$. So, if

$$
\boldsymbol{\delta}_{i}=-\delta_{i} \frac{\boldsymbol{k}_{i}}{\left|\boldsymbol{k}_{i}\right|}, \quad i=1,2
$$

where $\boldsymbol{k}_{i}$ are the wave vectors perpendicular to the wave crests, then we get 


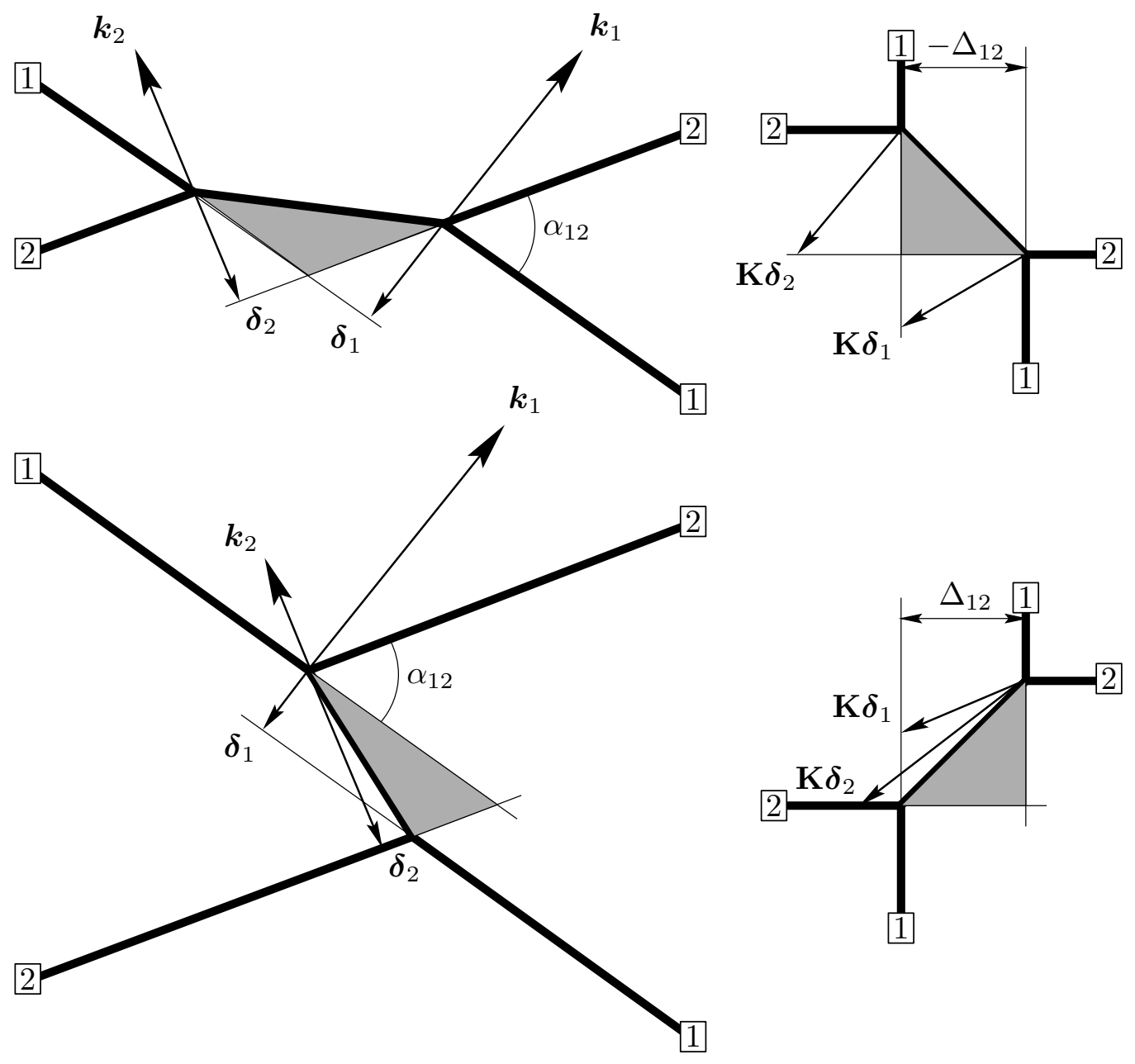

Fig. 12. Inverse relations between the real and phase pattern sets for the negative (upper figure) and positive (lower figure) phase shift cases.

from $\left(\mathbf{K} \boldsymbol{\delta}_{i}\right)_{i}=\boldsymbol{k}_{i}^{\mathrm{T}} \boldsymbol{\delta}_{i}=-\left|\Delta_{12}\right|$ the following two equations

$$
\delta_{i}\left|\boldsymbol{k}_{i}\right|=\left|\Delta_{12}\right|, \quad i=1,2,
$$

which relates the measured wave shifts to the parameters in the wave vectors.

Relations for relative angles: Since the vectors $\boldsymbol{k}_{1}$ and $\boldsymbol{k}_{2}$ are perpendicular to the wave crests, the relative angle between the wave crests is equal to the angle between the wave vectors:

$$
\cos \alpha_{12}=\frac{\boldsymbol{k}_{1}^{\mathrm{T}} \boldsymbol{k}_{2}}{\left|\boldsymbol{k}_{1}\right|\left|\boldsymbol{k}_{2}\right|} .
$$

Completing the set of equations for the KP case: The KP equation is assumed to describe the motion of waves traveling mainly in one direction, in the direction of $x$-axis for the given KP equation (5). So, it is natural and with no restriction of generality, to put the $x$-axis in the middle of the 
wave vectors $\boldsymbol{k}_{1}$ and $\boldsymbol{k}_{2}$, so that

$$
0<\frac{\nu_{1}}{\mu_{1}}=-\frac{\nu_{2}}{\mu_{2}}<1
$$

with $\mu_{1}>0, \mu_{2}>0$. This equation together with equations (21) and (22) form the complete system of equations for determining the four unknown parameters $\mu_{1}, \mu_{2}, \nu_{1}, \nu_{2}$.

\subsection{Solving the system for the KP model}

For the KP equation the phase shift parameter of the two-soliton solution reads (see Section 4)

$$
\Delta_{12}=-\ln \frac{\rho^{2}-\left(\mu_{1}-\mu_{2}\right)^{2}}{\rho^{2}-\left(\mu_{1}+\mu_{2}\right)^{2}},
$$

where, using (23), $\rho=\frac{\nu_{1}}{\mu_{1}}-\frac{\nu_{2}}{\mu_{2}}=2 \frac{\nu_{1}}{\mu_{1}}$. So, we have $\nu_{1}=\frac{1}{2} \rho \mu_{1}, \nu_{2}=-\frac{1}{2} \rho \mu_{2}$, and consequently, $\left|\boldsymbol{k}_{i}\right|=\mu_{i} \sqrt{1+\rho^{2} / 4}, i=1,2$. Equations (21) give now $\delta_{1} \mu_{1}=$ $\delta_{2} \mu_{2}$ which is used in eliminating one of the parameters, say $\mu_{2}=\mu_{1} \delta_{1} / \delta_{2}$. Note also that $\rho=2 \nu_{1} / \mu_{1}=2 \tan \frac{1}{2} \alpha_{12}$ (this is the relation for the relative angle). Taking into account all these relations, we obtain from (21) and (24) an equation for one unknown parameter $\mu_{1}$ :

$$
\mu_{1} \delta_{1} \sqrt{1+\rho^{2} / 4}=\left|\ln \frac{\delta_{2}^{2} \rho^{2}-\left(\delta_{2}-\delta_{1}\right)^{2} \mu_{1}^{2}}{\delta_{2}^{2} \rho^{2}-\left(\delta_{2}+\delta_{1}\right)^{2} \mu_{1}^{2}}\right| .
$$

In general, Eq. (25) has three solutions: one trivial $\left(\mu_{1}=0\right)$ and two nontrivial (if to assume $\rho \neq 0$ ) as illustrated in Fig. 13. It shows graphs of the 1.h.s. and r.h.s. of Eq. (25). Clearly, the nontrivial solutions are always separated. It turns out that the solution of (25) belonging to the interval $\left(0, \delta_{2} \rho /\left(\delta_{1}+\delta_{2}\right)\right)$ corresponds to the negative phase shift case while the other solution $\left(\mu_{1}>\delta_{2} \rho /\left|\delta_{2}-\delta_{1}\right|\right)$ corresponds to the positive phase shift case. So, we have the following equations

$$
\mu_{1} \delta_{1} \sqrt{1+\rho^{2} / 4} \pm \ln \frac{\delta_{2}^{2} \rho^{2}-\left(\delta_{2}-\delta_{1}\right)^{2} \mu_{1}^{2}}{\delta_{2}^{2} \rho^{2}-\left(\delta_{2}+\delta_{1}\right)^{2} \mu_{1}^{2}}=0
$$

where "+" corresponds to the positive phase shift case.

In conclusion, for a specified phase shift, the solution of the inverse problem is unique and is generated by the solution of Eq. (26).

For the practical applicability in the laboratory the sensitivity of the constructed solution on the measured parameters is of importance. In our next 


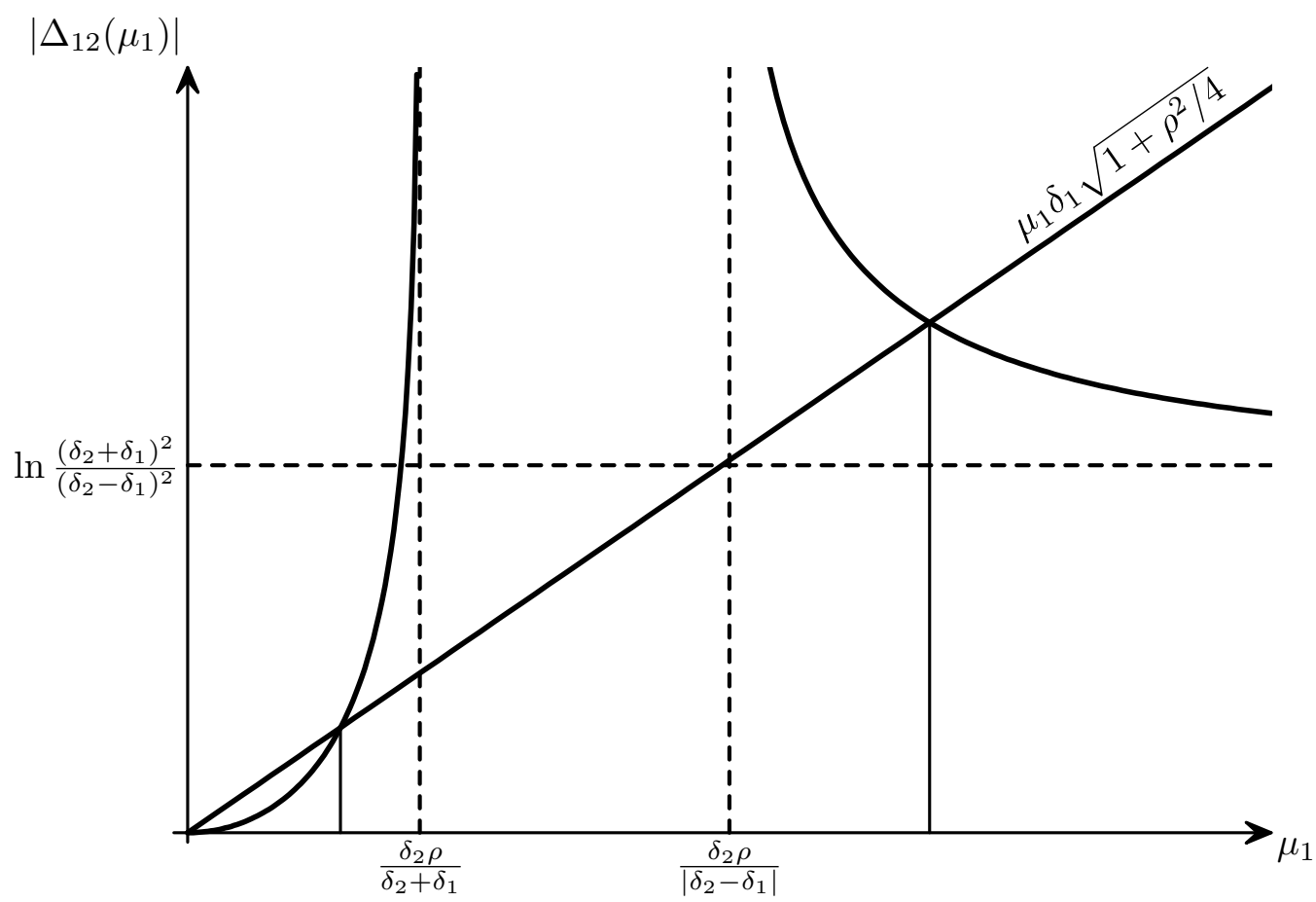

Fig. 13. Graphical representation of Eq. (25) shows the existence of two nontrivial solutions. Here $\left|\Delta_{12}\left(\mu_{1}\right)\right|=$ r.h.s. of $(25)$.

paper this sensitivity will be investigated in detail (see [13]).

\section{Discussion}

In this paper we considered the inverse problem of finding the amplitudes of interacting waves from the geometry of their interaction pattern, which is, for example, observable from optical measurements. We showed that this problem has a unique solution for the two wave interaction in the case the model is the KP equation. The sensitivity of the solution for errors in parameters that have to be determined from experiments has been investigated in detail but is not presented here because of limitation of space (see [13]). Furthermore, our approach has been general and can without modification be applied to solve the inverse problem for waves described by other KdV-type equations.

The direct problem of constructing the wave pattern of interacting solitons with known values of the parameters, demonstrated in this paper for two solitons, can be extended to the interaction of more solitons, see [14, 15], and will be the subject of a subsequent paper. However, at this moment it is not clear if the inverse problem for more than two interacting solitons can be solved, in particular when only a part of the interaction pattern is considered (which would be the information from a photographic registration), or when 
a time record of point measurements from such a part would be available.

The restriction of the present paper to consider solitons instead of periodic wave trains (cnoidal waves), leads to new questions. Since most wave phenomena in nature are, or appear to be, quasi-periodic, and since the KdV-type of equations support quasi-periodic soliton solutions that can be expressed in terms of the Riemann theta-functions, another challenge would be to solve the direct and inverse problem for quasi-periodic waves. Again the situation for two waves seems clear: Hammack et al. [2] have found that for two periodic waves the interaction pattern is hexagonal, and so the direct problem has a straightforward solution. However, when considering three or more interacting periodic solitons, the direct problem, and even more so the inverse problem, is much more difficult; the phase pattern needs to be determined and ways to construct it in an algorithmic way have to be found.

\section{Acknowledgements}

The inverse problem described in Section 6 has also been a topic in the Research Workshop "Aspects of Computational Fluid Dynamics" executed at Institut Teknologi Bandung, Indonesia, June 9 - August 1, 1997. One of us, EvG, would like to thank the participants, in particular Andi Jamaluddin, Ipung Yuwono, Jafar and Ong Chee Tiong for fruitful discussions.

Part of this research is sponsored by the Royal Netherlands Academy of Arts and Sciences in the programme for scientific cooperation NetherlandsIndonesia. One of the authors (PP) acknowledges the Estonian Science Foundation for financial support (grant 2631).

\section{A Proof of the formula (12)}

Let $\boldsymbol{n} \in \mathbb{Z}^{g}, I \subset \mathbb{Z}^{g}$, and $|I|<\infty$. Then

$$
\Theta=\sum_{\boldsymbol{m} \in I} A_{\boldsymbol{m}} e^{\boldsymbol{m}^{\mathrm{T}} \boldsymbol{\varphi}}=e^{-\boldsymbol{n}^{\mathrm{T}} \boldsymbol{\varphi}} \sum_{\boldsymbol{m} \in I} A_{\boldsymbol{m}} e^{(\boldsymbol{m}+\boldsymbol{n})^{\mathrm{T}} \boldsymbol{\varphi}}=e^{-\boldsymbol{n}^{\mathrm{T}} \boldsymbol{\varphi}} \Theta^{\prime}
$$

where $\Theta^{\prime}$ and $\Theta$ are equivalent because of the gauge invariance of $L$.

First, we consider the limit process $\varphi_{i} \rightarrow-\infty$. Here we choose index vector $\boldsymbol{n}$ such that (i) $(\boldsymbol{m}+\boldsymbol{n})_{i} \geqslant 0$ for all $\boldsymbol{m} \in I$ and (ii) there exists at least one $\boldsymbol{m} \in I$ such that $(\boldsymbol{m}+\boldsymbol{n})_{i}=0$. This choice of $\boldsymbol{n}$ ensures that $\lim _{\varphi_{i} \rightarrow-\infty} \Theta^{\prime}$ 
exists and is nonzero. Let us denote this limit by $\Theta^{\prime \prime}$ and find

$$
\Theta^{\prime \prime}=\lim _{\varphi_{i} \rightarrow-\infty} \Theta^{\prime}=\lim _{\varphi_{i} \rightarrow-\infty} \sum_{\boldsymbol{m} \in I} A_{\boldsymbol{m}} e^{(\boldsymbol{m}+\boldsymbol{n})^{\mathrm{T}} \boldsymbol{\varphi}}=\sum_{\substack{\boldsymbol{m} \in I \\(\boldsymbol{m}+\boldsymbol{n})_{i}=0}} A_{\boldsymbol{m}} e^{(\boldsymbol{m}+\boldsymbol{n})^{\mathrm{T}} \boldsymbol{\varphi}} .
$$

The r.h.s. of (12) becomes now

$$
L\left[\Theta^{\prime \prime}\right]=2 \frac{\Theta_{x x}^{\prime \prime} \Theta^{\prime \prime}-\Theta_{x}^{\prime \prime 2}}{\Theta^{\prime \prime 2}}
$$

where

$$
\begin{aligned}
\Theta_{x}^{\prime \prime} & =\sum_{\substack{\boldsymbol{m} \in I \\
(\boldsymbol{m}+\boldsymbol{n})_{i}=0}} A_{\boldsymbol{m}}(\boldsymbol{m}+\boldsymbol{n})^{\mathrm{T}} \boldsymbol{\mu} e^{(\boldsymbol{m}+\boldsymbol{n})^{\mathrm{T}} \boldsymbol{\varphi}} \\
\Theta_{x x}^{\prime \prime} & =\sum_{\substack{\boldsymbol{m} \in I \\
(\boldsymbol{m}+\boldsymbol{n})_{i}=0}} A_{\boldsymbol{m}}\left((\boldsymbol{m}+\boldsymbol{n})^{\mathrm{T}} \boldsymbol{\mu}\right)^{2} e^{(\boldsymbol{m}+\boldsymbol{n})^{\mathrm{T}} \boldsymbol{\varphi}}
\end{aligned}
$$

Note that $\Theta^{\prime \prime}$ does not depend on $\varphi_{i}$ but it is still correct to apply the operator $L$ (that includes partial derivatives with respect to $\varphi_{i}$ ) to it: the corresponding terms in $L\left[\Theta^{\prime \prime}\right]$ become just zeros.

Let us calculate the 1.h.s. of (12) with theta-function $\Theta^{\prime}$. First

$$
L\left[\Theta^{\prime}\right]=2 \frac{\Theta_{x x}^{\prime} \Theta^{\prime}-\Theta_{x}^{\prime 2}}{\Theta^{\prime 2}}
$$

where

$$
\begin{aligned}
\Theta_{x}^{\prime} & =\sum_{\boldsymbol{m} \in I} A_{\boldsymbol{m}}(\boldsymbol{m}+\boldsymbol{n})^{\mathrm{T}} \boldsymbol{\mu} e^{(\boldsymbol{m}+\boldsymbol{n})^{\mathrm{T}} \boldsymbol{\varphi}} \\
\Theta_{x x}^{\prime} & =\sum_{\boldsymbol{m} \in I} A_{\boldsymbol{m}}\left((\boldsymbol{m}+\boldsymbol{n})^{\mathrm{T}} \boldsymbol{\mu}\right)^{2} e^{(\boldsymbol{m}+\boldsymbol{n})^{\mathrm{T}} \boldsymbol{\varphi}}
\end{aligned}
$$

Since $\Theta^{\prime}, \Theta_{x}^{\prime}$, and $\Theta_{x x}^{\prime}$ remain finite as $\varphi_{i} \rightarrow-\infty$ then the limit over $L\left[\Theta^{\prime}\right]$ can be taken separately over $\Theta^{\prime}, \Theta_{x}^{\prime}$, and $\Theta_{x x}^{\prime}$. The result is exactly $L\left[\Theta^{\prime \prime}\right]$ in (A.1).

Similar arguments give formula (12) also for the limit process $\varphi_{i} \rightarrow+\infty$. Here the index vector $\boldsymbol{n}$ is chosen such that (i) $(\boldsymbol{m}+\boldsymbol{n})_{i} \leqslant 0$ for all $\boldsymbol{m} \in I$ and (ii) $\exists \boldsymbol{m} \in I:(\boldsymbol{m}+\boldsymbol{n})_{i}=0$. 


\section{B Derivation of formulas}

Equations (16) and (17) follow from

$$
\begin{aligned}
\lim _{\varphi_{1} \rightarrow-\infty} U\left(\varphi_{1}, \varphi_{2}\right) & =\lim _{\varphi_{1} \rightarrow-\infty} L\left[\Theta\left(\varphi_{1}, \varphi_{2}\right)\right] \\
& =L\left[\lim _{\varphi_{1} \rightarrow-\infty}\left(1+e^{\varphi_{1}}+e^{\varphi_{2}}+A_{12} e^{\varphi_{1}+\varphi_{2}}\right)\right] \\
& =L\left[1+e^{\varphi_{2}}\right]=U_{1}\left(\varphi_{2}\right), \\
\lim _{\varphi_{1} \rightarrow+\infty} U\left(\varphi_{1}, \varphi_{2}\right) & =\lim _{\varphi_{1} \rightarrow+\infty} L\left[e^{\varphi_{1}}\left(e^{-\varphi_{1}}+1+e^{\varphi_{2}-\varphi_{1}}+A_{12} e^{\varphi_{2}}\right)\right] \\
& =L\left[\lim _{\varphi_{1} \rightarrow+\infty}\left(e^{-\varphi_{1}}+1+e^{\varphi_{2}-\varphi_{1}}+A_{12} e^{\varphi_{2}}\right)\right] \\
& =L\left[1+A_{12} e^{\varphi_{2}}\right]=U_{1}\left(\varphi_{2}+\ln A_{12}\right) .
\end{aligned}
$$

Note that we must use the gauge invariance (11) before we can apply commutability property (12) of the operators $L$ and lim. Analogously the limits for $\varphi_{2} \rightarrow \pm \infty$ are found.

For $A_{12}=0$ we have

$$
\begin{aligned}
\lim _{\substack{\varphi_{1}+\varphi_{2} \rightarrow+\infty \\
\left|\varphi_{1}-\varphi_{2}\right|<\infty}} U\left(\varphi_{1}, \varphi_{2}\right) \\
=\lim _{\substack{\varphi_{1}+\varphi_{2} \rightarrow+\infty \\
\left|\varphi_{1}-\varphi_{2}\right|<\infty}} L\left[e^{\frac{\varphi_{1}+\varphi_{2}}{2}}\left(e^{-\frac{\varphi_{1}+\varphi_{2}}{2}}+e^{\frac{\varphi_{1}-\varphi_{2}}{2}}+e^{-\frac{\varphi_{1}-\varphi_{2}}{2}}\right)\right] \\
=L\left[\lim _{\substack{\varphi_{1}+\varphi_{2} \rightarrow+\infty \\
\left|\varphi_{1}-\varphi_{2}\right|<\infty}}\left(e^{-\frac{\varphi_{1}+\varphi_{2}}{2}}+2 \cosh \left(\varphi_{1}-\varphi_{2}\right)\right)\right] \\
=L\left[\cosh \left(\varphi_{1}-\varphi_{2}\right)\right]=U_{1}\left(\varphi_{1}-\varphi_{2}\right) .
\end{aligned}
$$

For $A_{12} \rightarrow+\infty$ we have

$$
\begin{aligned}
\lim _{A_{12} \rightarrow+\infty} U\left(\varphi_{1}, \varphi_{2}\right) & =\lim _{A_{12} \rightarrow+\infty} L\left[1+e^{\varphi_{1}}+e^{\varphi_{2}}+A_{12} e^{\varphi_{1}+\varphi_{2}}\right] \\
& =\lim _{A_{12} \rightarrow+\infty} L\left[\left(1+e^{\varphi_{1}}+e^{\varphi_{2}}\right) / A_{12}+e^{\varphi_{1}+\varphi_{2}}\right] \\
& =L\left[e^{\varphi_{1}+\varphi_{2}}\right]=0 .
\end{aligned}
$$


If $\Theta$ is defined by (15) then

$$
\begin{aligned}
& L\left[\Theta\left(\varphi_{1}, \varphi_{2}\right)\right]=L\left[e^{\frac{\varphi_{1}+\varphi_{2}}{2}}\left(e^{-\frac{\varphi_{1}+\varphi_{2}}{2}}+e^{\frac{\varphi_{1}-\varphi_{2}}{2}}+e^{-\frac{\varphi_{1}-\varphi_{2}}{2}}+A_{12} e^{\frac{\varphi_{1}+\varphi_{2}}{2}}\right)\right] \\
& \quad=L\left[2 \cosh \frac{\varphi_{1}-\varphi_{2}}{2}+\sqrt{A_{12}}\left(e^{\left.\left.-\frac{\varphi_{1}+\varphi_{2}}{2}-\ln \sqrt{A_{12}}+e^{\frac{\varphi_{1}+\varphi_{2}}{2}+\ln \sqrt{A_{12}}}\right)\right]}\right.\right. \\
& \quad=L\left[\cosh \frac{\varphi_{1}-\varphi_{2}}{2}+\sqrt{A_{12}} \cosh \frac{\varphi_{1}+\varphi_{2}+\ln A_{12}}{2}\right] .
\end{aligned}
$$

\section{References}

[1] J. Hammack, N. Scheffner, and H. Segur. Two-dimensional periodic waves in shallow water. J. Fluid Mech., 209:567-589, 1989.

[2] J. Hammack, D. McCallister, N. Scheffner, and H. Segur. Twodimensional periodic waves in shallow water. Part 2. Asymmetric waves. J. Fluid Mech., 285:95-122, 1995.

[3] B. Grammaticos, A. Ramani, and J. Hietarinta. A search for integrable bilinear equations: The Painlevé approach. J. Math. Phys., 31(11):25722578, November 1990.

[4] R. Hirota. Direct methods in soliton theory. In R. K. Bullough and P. J. Caudrey, editors, Solitons, pages 157-176. Springer, Berlin, 1980.

[5] C. S. Gardner, J. M. Greene, M. D. Kruskal, and R. M. Miura. Kortewegde Vries equation and generalizations. VI. Methods for exact solution. Comm. Pure Appl. Math., XXVII:97-133, 1974.

[6] T. Yoneyama. The Korteweg-de Vries two-soliton solution as interacting two single solitons. Prog. Theor. Phys., 71(4):843-846, April 1984.

[7] P. F. Hodnett and T. P. Moloney. On the structure during interaction of the two-soliton solution of the Korteweg-de Vries equation. SIAM J. Appl. Math., 49(4):1174-1187, August 1989.

[8] A. C. Bryan and A. E. G. Stuart. On the dynamics of soliton interactions for the Korteweg-de Vries equation. Chaos, Solitons $\&$ Fractals, 2(5):487491, 1992.

[9] R. Hirota and M. Ito. Resonance of solitons in one dimension. J. Phys. Soc. Jpn., 52(3):744-748, March 1983.

[10] R. J. LeVeque. On the interaction of nearly equal solitons in the KdV equation. SIAM J. Appl. Math., 47(2):254-262, April 1987.

[11] A. C. Newell. Solitons in Mathematics and Physics. Society for Industrial and Applied Mathematics, 1985.

[12] H. Segur and A. Finkel. An analytical model of periodic waves in shallow water. Stud. Appl. Math., 73:183-220, 1985.

[13] P. Peterson and E. van Groesen. Sensitivity of the wave crest problem. Wave Motion, 1999. (Submitted).

[14] P. Peterson. I. Interaction of KdV-type solitons in phase variables. Research Report Mech 185/98, Inst. of Cybernetics, 1998.

[15] P. Peterson. II. Interaction of KdV-type solitons in real space-time variables. Research Report Mech 180:398, Inst. of Cybernetics, 1998. 